\title{
Materials Science and Engineering $C$
}

https://doi.org/10.1016/j.msec.2020.111695

Received 15 September 2020; Received in revised form 26 October 2020; Accepted 30 October 2020

Available online 4 November 2020

\section{Viscoelastically active sutures - A stitch in time?}

\author{
Louise A. France*, Kevin S. Fancey \\ Department of Engineering, University of Hull, HU6 7RX, UK
}

\begin{abstract}
We present results to show that a commercially available polypropylene suture filament (Ethicon Prolene), following annealing and tensile creep can, after creep load removal, release viscoelastically stored energy over a period of several weeks. Specifically, over $0.1-1000 \mathrm{~h}$, the suture undergoes a time-dependent contraction of $\sim 4 \%$ and, following a short recovery time $(\sim 3 \mathrm{~min})$ to a fixed strain, produces a progressively increasing recovery force of $\sim 0.1-1 \mathrm{~N}$. We suggest that this time-dependent energy release may facilitate wound healing by the action of viscoelastically induced mechanotransduction (VIM). Moreover, our recent (published) findings have led to evidence of reduced hydrophobicity from viscoelastically recovering polymeric filaments and speculation that this may emanate from the long-term release of electric charges. Thus, we propose that the latter may enhance the VIM mechanism. In this paper, we report on the direct detection of these charges and the first findings from an investigation involving the presence of cell cultures on Prolene samples that are (i) viscoelastically recovering, (ii) annealed only and (iii) in as-received condition. From (i), the results demonstrate a significant increase in cell motility, with migration towards the suture, compared to (ii) and (iii). This suggests greater stimulation of the wound healing process, an effect which is expected to continue for the duration of the viscoelastic recovery period.
\end{abstract}

Keywords: Wound healing; Sutures; Mechanotransduction; Viscoelasticity; Cell motility; Electric charge.

\footnotetext{
* Corresponding author. Tel.: + 441482466375.

Email:1.france@hull.ac.uk (L.A. France).
} 


\section{Introduction}

Sutures are routinely used to assist the healing of wounds, since they can provide mechanical support to the wound area, reduce bleeding, infection risks and minimise scarring. Sutures (absorbable and non-absorbable) are commonplace, and in recent years, these have been augmented by the incorporation of coating treatments to reduce microbial activity. Moreover, emerging trends include the introduction of drug-eluting and stem cell-seeded sutures [1]. In addition to developments in "passive" sutures, there has been an upsurge of interest in "smart" sutures. Principally, these exploit the use of shape-memory materials to enable self-tightening and self-knotting for deep wound closures, especially in key-hole surgery [1-4], but there has also been a growing interest in electronic sutures, with monitoring and actuation capabilities for wound management $[1,5,6]$; i.e. truly smart sutures.

To date, the possibilities for exploiting mechanical activity in a suture material have received some attention, but only in two areas. First, as mentioned above, are materials that possess shape-memory (temperature-sensitive) characteristics to enable self-knotting. The second area exploits the development of materials with low elastic moduli but sufficient mechanical strength for soft tissue wound closures (e.g. midline laparotomy) [1,7]. In this paper, we report the first findings from a novel material treatment technique focused on the time-dependent mechanical characteristics of a commercially available suture. The aim of this technique is to promote an improved wound healing response, by the suture releasing viscoelastically stored energy over a period of several weeks.

\section{Background}

\subsection{Viscoelastic activity - mechanotransduction}

Viscoelasticity is perhaps most often associated with undesirable behaviour, such as the creep or stress relaxation problems that may be encountered within engineering structures. Nevertheless, the phenomenon can be exploited to good effect, one example being in the development of viscoelastically prestressed polymeric matrix composites (VPPMCs). A VPPMC is produced by subjecting strong polymeric fibres to a tensile load so that they undergo viscoelastic creep; then, following load removal, the (loose) fibres are moulded within a resin matrix. On matrix solidification, the fibres continue to attempt contraction due to their viscoelastically stored energy; this creates compressive stresses within the matrix which are counterbalanced by residual tension within the fibres [8]. VPPMCs based on nylon 6,6, UHMWPE and regenerated cellulose fibres have demonstrated mechanical property improvements of 20-60\% compared with control (no prestress) counterparts [8-10] and additionally, may offer benefits in the development of novel structures such as shape-changing components [11].

The knowledge gained from the above work leads one to consider whether such viscoelastic behaviour could be applied to areas other than engineering composites. Its applicability to sutures and other medical devices (e.g. scaffolds) is one possibility. Mechanotransduction, i.e. the mechanical stimulation of cells to elicit electrochemical activity is a mechanism that can improve wound healing [12]. Given that most commercially available sutures are polymeric materials, they should have the capability for storing viscoelastic energy. Thus it may be possible to treat a suture, prior to wound repair, so that the suture undergoes a small, progressive lengthwise contraction 
throughout the healing period; i.e. the wound would be subjected to viscoelastically induced mechanotransduction (VIM).

\subsection{Viscoelastic activity - electric charge phenomena}

In addition to viscoelastic activity producing a mechanical response, recent investigative work on VPPMCs has led to speculation of the occurrence of another phenomenon. By analysis with the scanning electron microscope mirror effect, it was found that $\sim 30 \%$ fewer electric charges were trapped at the fibre-matrix interfaces of VPPMC samples compared with control counterparts. From this, it was proposed that viscoelastic activity within the fibres could influence electric charge interactions at the fibre-matrix interface regions [13].

It is known that mechanical stresses, when applied to an insulating material, lead to an injection of electric charges [14] and, for intermediately disordered materials such as polymers, low mobility leads to a strong localisation (trapping) of these charges [15], thus enabling the storage of significant polarisation energy $(\sim 5 \mathrm{eV}$ or more per trapped charge) [16]. Therefore, this may be expected to occur when polymeric fibres are subjected to creep for VPPMC production. It was suggested in [13] that viscoelastic recovery mechanisms within the fibres gradually release these charges. Moreover, viscoelastic recovery from fibres investigated for VPPMC production is known to be a long-term phenomenon which, even at an ambient temperature of $50{ }^{\circ} \mathrm{C}$, exceeds 20 years [17]. The recovery is suggested to occur through the time-dependent triggering of molecular jumps, with longer term activity being represented by sites triggered through very long time constants $[18,19]$.

From the above considerations, it was speculated in [13] that the dielectric and viscoelastic properties of a polymer correspond to the same movement of molecular chains, so that the release of trapped charges may concur with the triggering of molecular jumps during viscoelastic activity; i.e. electric charges are released over the same timescale as viscoelastic recovery.

\subsection{Viscoelastic activity - enhanced suture performance?}

The potential success of VIM to elicit improved healing (Section 2.1), depends on the suture undergoing sufficient progressive lengthwise contraction during the wound repair period $(\sim 1000 \mathrm{~h})$. The magnitude and duration of this contraction is determined by the viscoelastically generated recovery strain and force output produced after the suture has been subjected to a period of tensile creep. Subsequently, the treated suture can be applied to the wound region.

Fig. 1(a) represents the general strain-time creep and recovery characteristics of a viscoelastic material. Here, $\varepsilon_{\mathrm{c}}(t)$ is the (time dependent) creep strain, $\varepsilon_{\mathrm{i}}$ and $\varepsilon_{\mathrm{e}}$ are the elastic strains following initial loading and load release respectively. An equation based on the Weibull or Kohlrausch-Williams-Watts (KWW) function [18] can be used to describe the time-dependent viscoelastic recovery strain $\varepsilon_{\mathrm{rvis}}(t)$, from a polymeric filament previously subjected to tensile creep: 


$$
\varepsilon_{\text {rvis }}(t)=\varepsilon_{\mathrm{r}}\left[\exp \left(-\left(\frac{t}{\eta_{\mathrm{r}}}\right)^{\beta_{\mathrm{r}}}\right)\right]+\varepsilon_{\mathrm{f}}
$$

Where $\eta_{\mathrm{r}}$ and $\beta_{\mathrm{r}}$ in the $\varepsilon_{\mathrm{r}}$ function represent the Weibull characteristic life and shape parameters respectively. Permanent strain resulting from viscous flow is represented by $\varepsilon_{\mathrm{f}}$. Similarly, the time-dependent viscoelastic recovery force, $F(t)$, from a filament recovering at a fixed strain, can be represented by [20]:

$$
F(t)=F_{v}\left[\exp \left(-\left(\frac{\Delta t}{\eta}\right)^{\beta}\right)-\exp \left(-\left(\frac{t}{\eta}\right)^{\beta}\right)\right]
$$

Where $\eta$ and $\beta$ are the associated Weibull parameters within the $F_{\mathrm{v}}$ function, $\Delta t$ is the time interval between releasing the creep stress and initiation of the recovery stress (Fig. 1(b)).

(a)

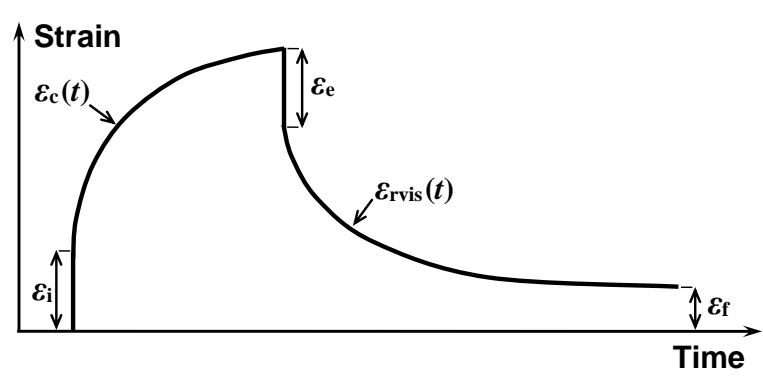

(b)

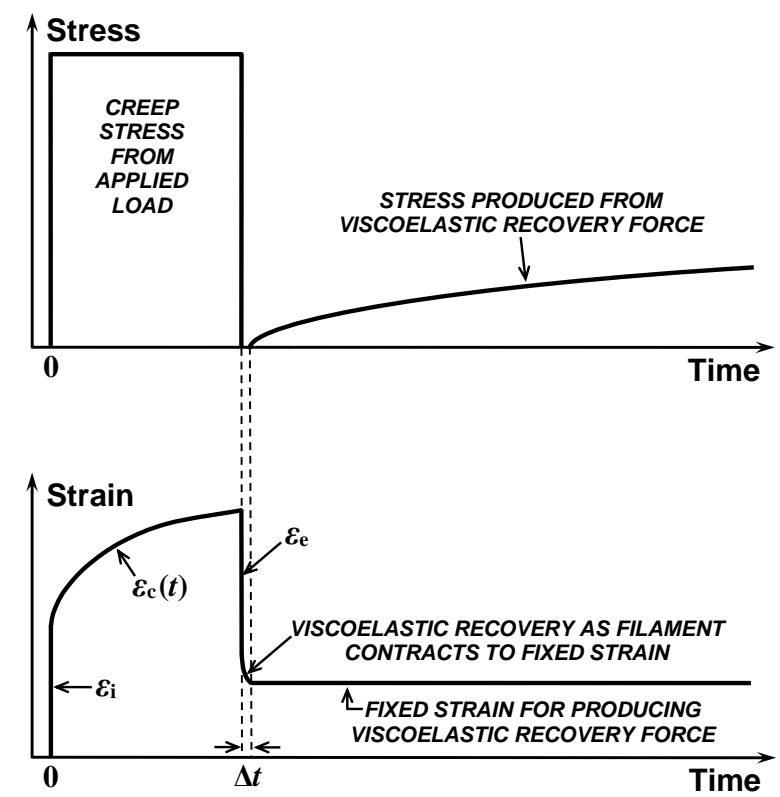

Fig. 1. (a) General strain-time creep and recovery characteristics of a viscoelastic filament; (b) schematic of the creep-recovery test cycle to evaluate the recovery force-time characteristics from a viscoelastic filament. 
Previous studies on the development of VPPMCs have shown that annealing the fibres prior to applying a creep load provides significant benefits to the magnitude and longevity of viscoelastic activity during recovery [8]. Thus, by subjecting suture filaments to a range of anneal and creep loading conditions, Eq. (1) and Eq. (2) may be used to assist in ascertaining the most appropriate values to maximise the possible effects from viscoelastic activity over the wound repair period.

Speculation over the possible long-term release of electric charges during viscoelastic recovery (Section 2.2) leads to consideration that this could affect hydrophobicity. For insulating materials, there is a strong correlation between the presence of electric charge and loss of hydrophobicity [21]. Thus, a viscoelastically recovering filament could be more hydrophilic than its control counterpart. A recent investigation involving the observation of water droplets on nylon monofilaments and fibres has indicated reduced hydrophobicity as a result of viscoelastic recovery effects $[22,23]$. This provides some evidence of the electric charge phenomenon; moreover, it is recognised that the presence of electric charges is of fundamental importance in biological processes, including wound healing [24]. Therefore, in addition to the purely mechanical aspects of VIM, a viscoelastically recovering suture may, through a progressive release of electric charges, provide further benefits to the wound healing process by influencing cell motility and migration.

\section{Materials and methods}

\subsection{Sample production and strain measurements}

Since this initial study was to evaluate the feasibility of VIM effects, a small number of creep-recovery strain runs were undertaken to determine a suitable viscoelastic recovery range from a readily available suture product. Therefore, the intention was not to seek optimum materials or viscoelastic treatment conditions here; the current work is a precursor to a more extensive study for such purposes.

Ethicon Prolene 2-0, a common thermoplastic (polypropylene-based) suture, was selected for this study. All suture samples were annealed, unconstrained, in a fanassisted oven at $120^{\circ} \mathrm{C}$. Following annealing, the suture was cut into two sample lengths, one designated as test, the other being control. The test sample was subjected to tensile creep whilst the control was positioned in close proximity to enable exposure to the same ambient conditions $\left(20-21^{\circ} \mathrm{C}, 30-60 \% \mathrm{RH}\right)$. For creep loading and strain measurement, the test sample was attached as a loop over the upper and lower bobbins of a simple stretching rig, the lower bobbin being fixed to a counterbalanced platform with weights to achieve a specified creep stress, as previously described [25]. Timedependent creep and recovery strain $\left(\varepsilon_{\text {rvis }}(t)\right.$ in Fig. 1(a)) values could be recorded by measuring the distance between two UV-illuminated ink marks on the suture (typically 200-250 mm apart) using a digital cursor with a precision of $\pm 0.01 \mathrm{~mm}$.

\subsection{Viscoelastic recovery force}

A bespoke rig with a force measurement transducer, as previously described [20], was used to evaluate the viscoelastic recovery force from a suture sample. Following a creep period on the stretching rig, the tensile stress was released and the loose suture loop (with bobbins) was transferred to the force measurement rig as quickly as possible. 
After a short period of time $\Delta t$ (Fig. 1(b)), the initially loose loop became progressively taut due to viscoelastic recovery. The resulting recovery force was monitored through the transducer at a fixed strain. All measurements were performed under ambient conditions of $20-21{ }^{\circ} \mathrm{C}$ and $30-60 \%$ RH.

\subsection{Suture hydrophobicity}

Section 2.3 highlighted a previous study of water droplets on nylon filaments, which indicated reduced hydrophobicity as a result of viscoelastic recovery effects [22, 23]. In the current study, an electrometer (Keithley Instruments Model 610B) was utilised to determine whether electric charge, believed to be responsible for reduced hydrophobicity, could be collected. Two suture samples, one being a viscoelastically recovering (test) sample, the other as control (annealed only), were each in turn inserted through the centre of a thin-walled copper tube (35 mm length, $12.5 \mathrm{~mm}$ internal diameter), connected to the electrometer. In each case, the sample ( $\sim 350 \mathrm{~mm}$ long) was suspended vertically, with the tube positioned mid-way. All metal supportive structures were earthed. Effectively, the arrangement of the suture sample within the electrically isolated tube was comparable to an air capacitor; hence the presence of a positive charge on the suture surface would, through electrostatic induction, cause the tube to become negatively charged, this being detected by the electrometer. The return path between the tube and electrometer (coaxial connection) was earthed.

Readings of charge accumulation from the electrometer were recorded over a $3 \mathrm{~h}$ period. Within this period, the test and control samples were repeatedly exchanged between successive $0.25 \mathrm{~h}$ measurement periods to ensure, as far as possible, consistent measurement conditions. The copper tube was discharged to earth between each measurement period.

\subsection{Cell culture}

Primary human dermal fibroblasts (HDFs) were maintained in high glucose Dulbecco's Modified Eagle's Medium (DMEM) with 2mM L-Glutamine (Sigma UK). This was supplemented with 10\% foetal bovine serum (FBS, Invitrogen, UK), $1 \%$ antibiotic and antimycotic (penicillin and streptomycin), and maintained in exponential growth at $37{ }^{\circ} \mathrm{C}, 5 \% \mathrm{CO}_{2}$, and $95 \%$ humidity.

\subsection{Cellular motility}

To examine cell motility in response to the treated sutures, a 24 well plate was set up to contain $2 \mathrm{~cm}$ lengths of the material samples ( $n=5$ per suture treatment). Suture samples were adhered to the well plate with silicone to prevent floatation and interference with the image capture techniques. The control wells contained only silicone adhesive, and subsequent wells contained either as-packaged, annealed-only or annealed and prestressed suture material.

HDFs were seeded at $1 \times 10^{4}$ cells/ $\mathrm{ml}$ and left until they had reached $30-40 \%$ confluence (approximately 3 hours). Each well contained $500 \mu 1$ supplemented cell culture medium to ensure visual acuity. 
A Phasefocus Livecyte system (Phasefocus, UK) was used to perform quantitative phase imaging (QPI), every 10 minutes for a total duration of 12 hours. Phase Focus Cell Analysis Toolbox software was used to segment and track individual cells [26], specifically measuring cell speed and meandering index. This was performed at 3 hours, 7 days, 14 days, 28 days and 42 days following the initiation of viscoelastic recovery.

\section{Results and discussion}

\subsection{Viscoelastic recovery properties}

Fig. 2 presents the viscoelastic recovery strain and force characteristics from suture samples. The data correlate well with Eqs. (1) and (2), which is consistent with other polymeric filament materials such as polyethylene $\{9]$, cellulose [10] and nylon $6,6[18,20]$.

From the tensile creep trials investigated in this initial study, Fig. 2(a) shows the recovery strain characteristics from two runs. For a fixed creep stress, these summarise our findings from a number of trials which demonstrated that annealing time, in addition to creep duration, has a significant role in the achievable recovery strain. Thus although the (undesirable) permanent strain $\varepsilon_{\mathrm{f}}$ from Run $2(7.4 \%)$ is greater than Run 1 $(6.0 \%)$, the available viscoelastic recovery strains $\left(\varepsilon_{\text {rvis }}(t)-\varepsilon_{\mathrm{f}}\right)$ over 0.1 to $1000 \mathrm{~h}$ are $3.8 \%$ and $2.6 \%$ respectively, i.e. the parameters for Run 2 would be expected to make a greater contribution to VIM effects.

Fig. 2(b) shows a plot of viscoelastically generated recovery force, from the creep parameters used for Run 2 in Fig. 2(a). Here, the suture loop became taut at $\sim 3$ min (i.e. $\sim 0.05 \mathrm{~h}$ for $\Delta t$ ), giving a force output, at a fixed $\varepsilon_{\text {rvis }}(t)$ strain of $\sim 12 \%$. The curve shows that the loop exceeds $2 \mathrm{~N}$ after $20 \mathrm{~h}$; i.e. a force of $1 \mathrm{~N}$ for a single suture filament. After $\sim 300 \mathrm{~h}$, the growth in force output diminishes, suggesting that the possibility of a decline (e.g. from stress relaxation) is being offset by long-term viscoelastic recovery mechanisms.

Evidence of an electric charge effect from suture samples undergoing viscoelastic recovery was demonstrated through charge collection by the electrometer arrangement (Section 3.3). Charge collection (negative polarity) over successive $0.25 \mathrm{~h}$ periods was no more than $\sim 10^{-3} \mu \mathrm{C}$; however, values within the first hour of recovery were 3-5 times greater for the test suture samples compared with control counterparts. Although this higher charge could be detected only over the first few hours, the findings suggest that a recovering suture possesses positive charge polarity. Clearly, a more detailed study is required, with an experimental arrangement that can provide greater sensitivity. Nevertheless, these preliminary results appear to concur with the prognosis that hydrophobicity is reduced during viscoelastic recovery. 
(a)

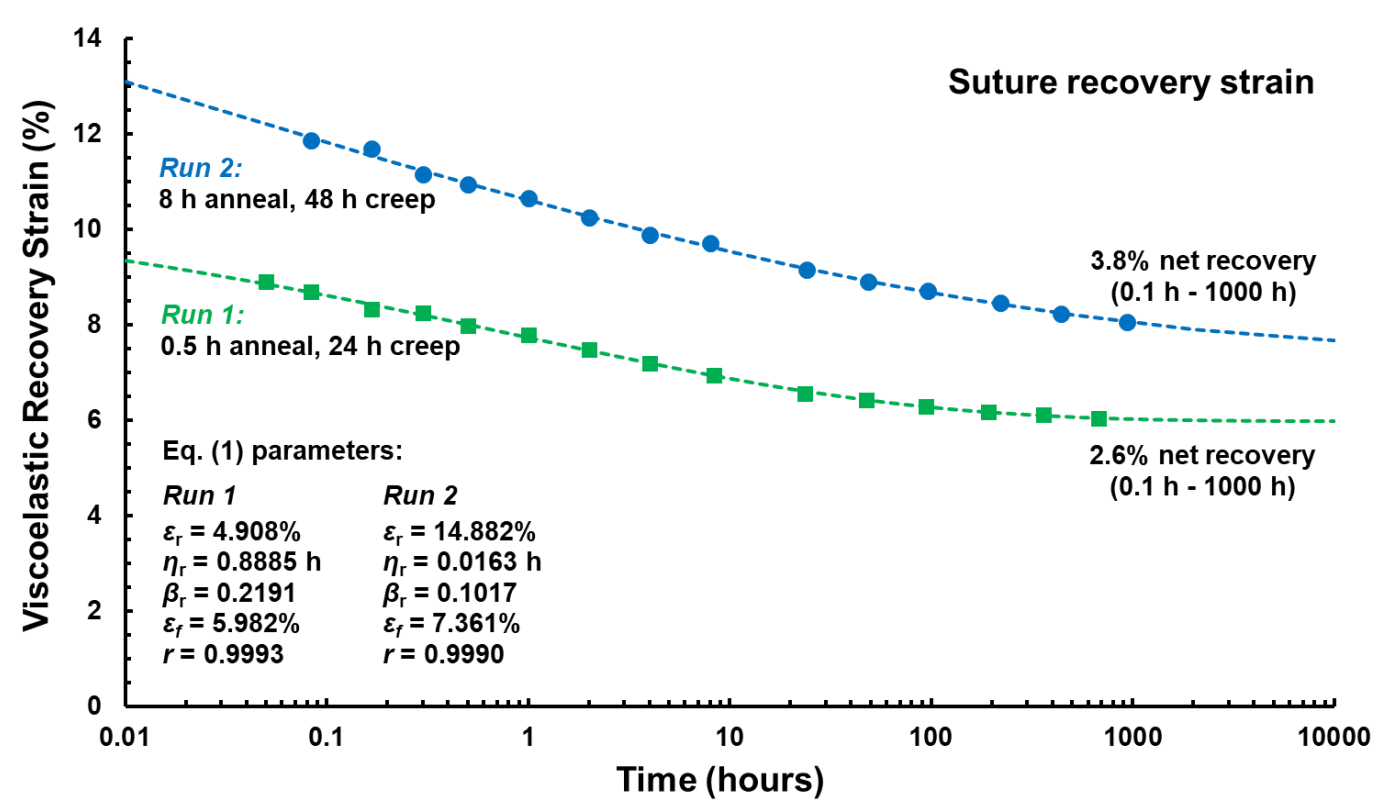

(b)

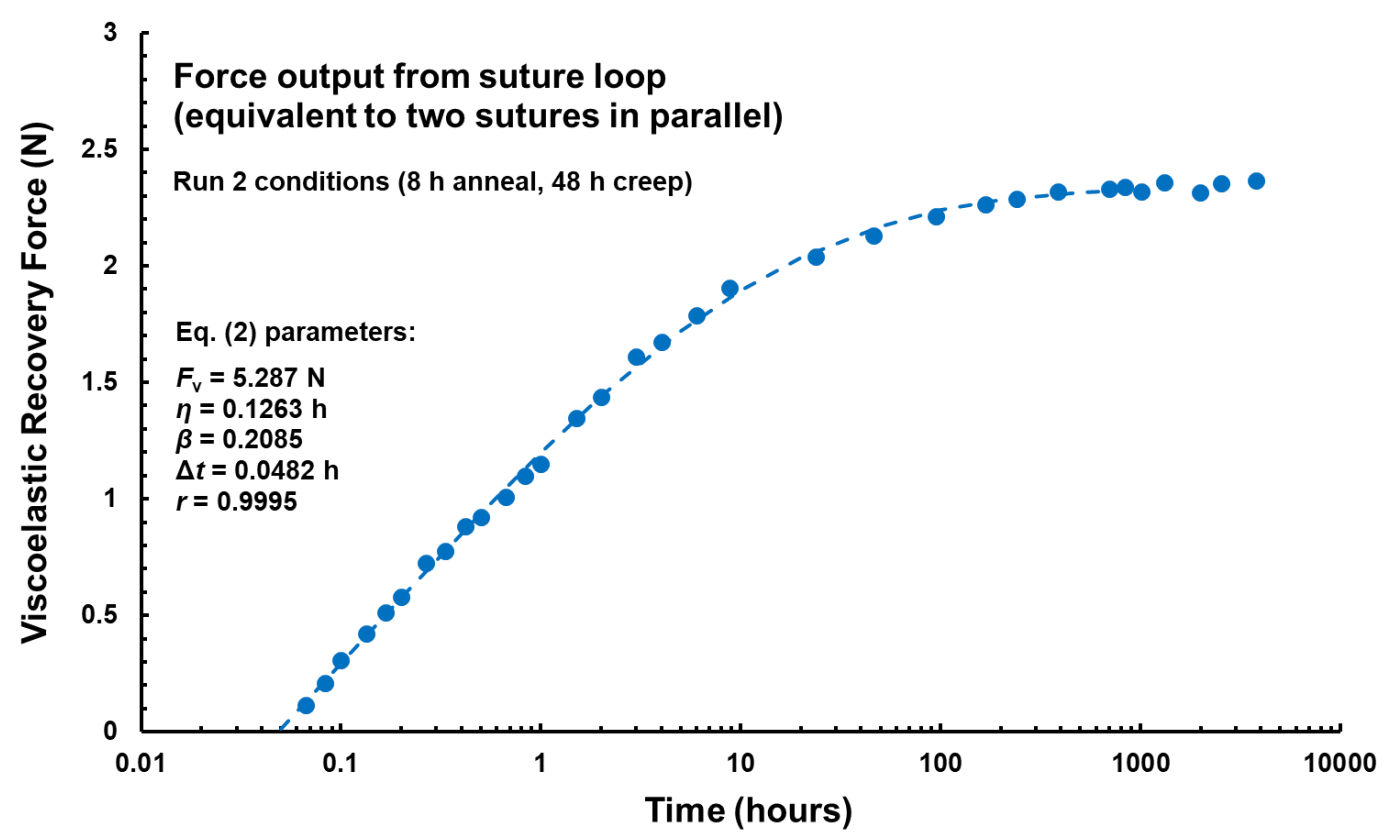

Fig. 2. Plots of viscoelastic recovery in terms of (a) strain and (b) force (fixed $\varepsilon_{\text {rvis }}(t)$ strain of $\sim 12 \%$ ); $r$ is the correlation coefficient for curve fitting to Eq. (1) or (2). For Runs 1 and 2, sutures were annealed at $120^{\circ} \mathrm{C}$ and creep stress was $135.3 \mathrm{MPa}$ at $20-21^{\circ} \mathrm{C}$.

\subsection{Cell motility}

Fig. 3 graphically represents the short-term response of HDFs when cultured with suture samples that were either prestressed (after annealing), annealed-only, or aspackaged (i.e. the commercially available product). Although there are notable variations within all sample pools $(n=5)$, a clear trend is visible. Control wells containing just the silicone adhesive had very little influence on the cell culture with an average meandering index of $0.270 \pm 0.005$ (SEM), and a mean cell speed of $0.140 \pm$ 
$0.003 \mu \mathrm{m} / \mathrm{min}$ (SEM), indicating that the results were repeatable at each time point. No statistical significance was observed between the as-packaged and annealed sutures although both gave a higher meandering index and cell speed than the control, suggesting that they had some influence on the cells. The prestressed sutures can, however, be seen to have the greatest influence on meandering index and cell speed, especially for the first 14 days, these being typically $\sim 0.7$ au and $\sim 0.35 \mu \mathrm{m} / \mathrm{min}$ respectively, i.e. approximately double the values from the as-packaged samples. At 28 and 42 days, Fig. 3 shows a progressive fall-off in these values, indicating that cell motility becomes less responsive to activity from the later stages of viscoelastic recovery in Fig. 2. These trends are more clearly indicated by plotting the motility parameters as a function of viscoelastic activity, the latter being represented by the $\varepsilon_{\mathrm{r}}$ function from Eq. (1), i.e. $\left(\varepsilon_{\text {rvis }}(t)-\varepsilon_{f}\right)$, as shown in Fig. 4. Here, Fig. 4 indicates a diminishing response as the viscoelastic recovery strain becomes less than $1 \%$. Thus, although the suture material and treatment procedures are not optimised, our findings clearly demonstrate that viscoelastic recovery mechanisms elicit a positive effect on cell motility.

(a)

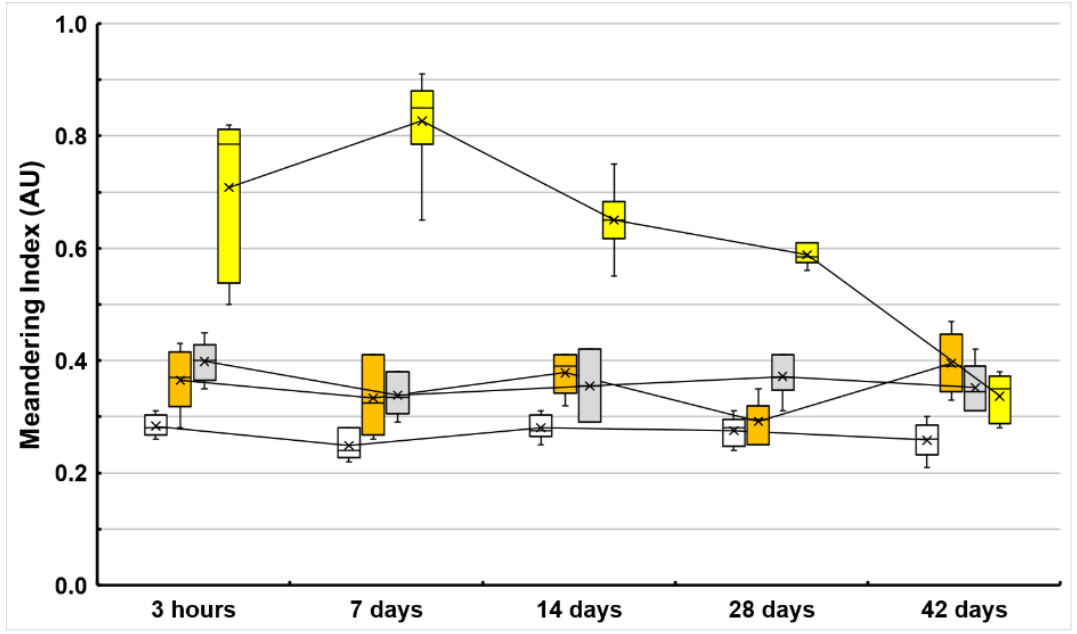

(b)

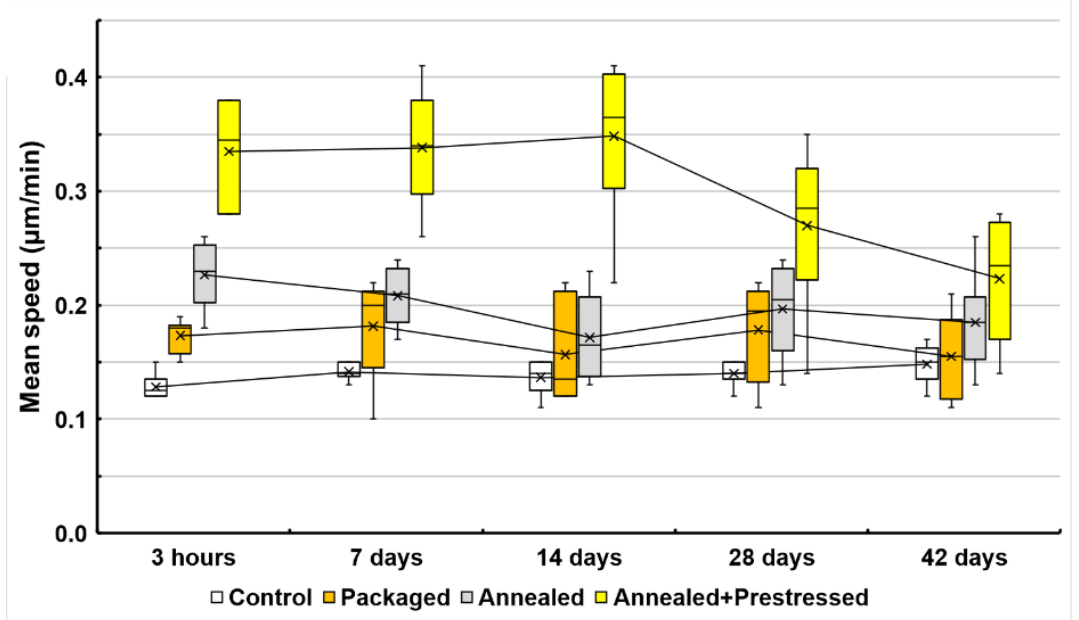

Fig. 3. Cell motility metrics calculated from the tracked cells in culture with either no suture (control), as-packaged, annealed, or prestressed sutures. Measurements taken at 3 hours, 7 days, 14 days, 28 days and 42 days: (a) is the average meandering index calculated per well $(n=5)$; (b) is the mean speed of cells calculated per well $(n=5)$. Error bars represent standard error of the mean. 


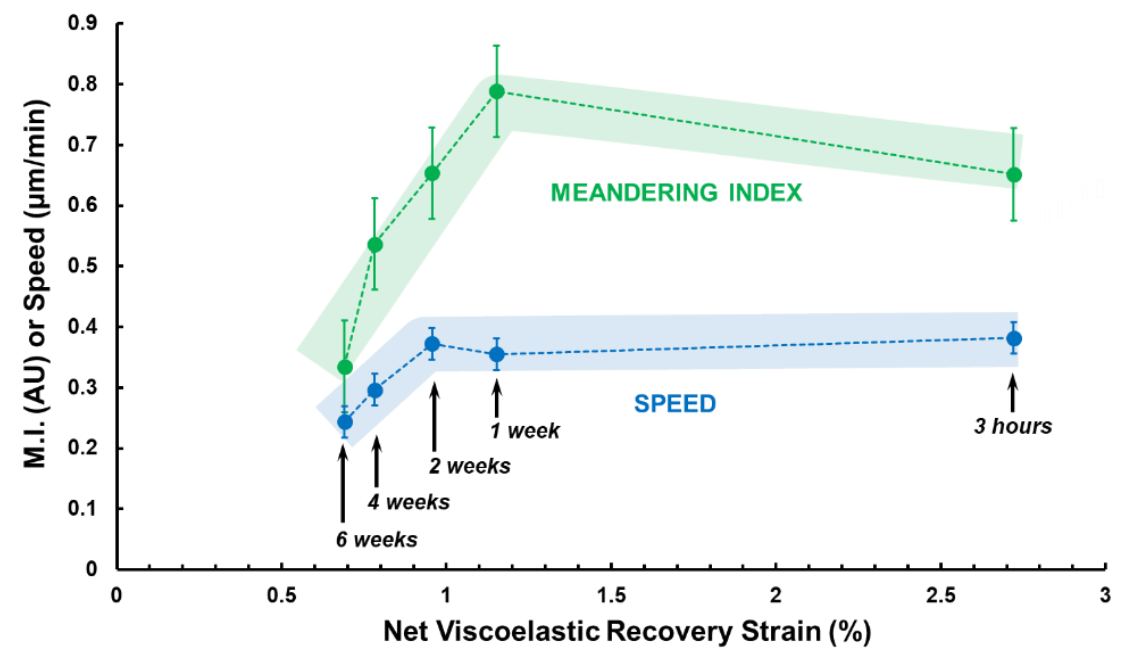

Fig. 4. Plot of motility parameters as a function of net viscoelastic recovery strain (i.e. $\left.\varepsilon_{\text {rvis }}(t)-\varepsilon_{\mathrm{f}}\right)$, indicating a decline in motility when the strain falls below $\sim 1 \%$; shaded regions represent the general trend. Error bars represent standard error of the mean.

The Livecyte system (Phasefocus) was able to detect the cells in the culture, and accurately track their movement using a simple vector analysis algorithm. This gave a value for the meandering index (arbitrary units). As the meandering index approached a value of unity, the cells could be seen to be tracking in a single direction; therefore, it was possible to deduce that the prestressed suture would be either attracting or repelling the cells. With the suggested change in hydrophobicity (the suture being positively charged, as discussed in Section 4.1), it is likely that the cells were tracking towards the suture sample. Studies have shown human dermal fibroblasts track towards anodes and away from cathodes when in a charged environment [27]. Whilst this is specific to cell type, and cannot be assumed for other cells involved in the wound healing process, it does appear to support the observation of directional movement in our culture system.

Standard tissue culture well plates (as used in this study) have a hydrophilic, negatively charged surface, as a means of encouraging rapid cell adhesion, and therefore proliferation [28]. Owing to the evenly distributed net charge, cells in basic culture typically exhibit random directionality due to lack of external stimulus, or in this case, an electric field [29]. In our system however, where there is a positively charged suture and a negatively charged culture surface, the cells appear to migrate in the direction of the positive charge; i.e. towards the suture, within the first 3 hours of recovery. This is not too dissimilar to the natural process of wound healing, in which breaking down the epithelial barrier leads to a flow of positive charge from surrounding tissues towards the wound centre, thereby triggering the accumulation of cells to initiate the wound healing process $[24,30]$. In contrast with the prestressed suture samples, the control, annealed and as-packaged equivalents all show notably lower meandering index values, indicating that cell movements were more randomly orientated; i.e. they were not responding to the presence of non-stressed suture samples.

Although classed as an independent metric, the mean cell speed has shown a parallel correlation with the meandering index in Figs. 3 and 4. When the cells are not influenced by the suture (control), they settle and adhere randomly in the well plate, and have a relatively slow motility rate. In contrast, cells in the well with the prestressed 
suture are seen to track in a single direction and at a much faster speed. The migration of fibroblasts to the wound site is one of the earliest and most critical stages of the wound healing response [31-32]. This is usually in response to immune cells being triggered, but is crucial to the laying down of the fibrous matrix within the wound site [33]. It is possible that the electric charge effect from prestressed sutures will assist in stimulating and guiding the recruitment of fibroblasts to the wound site, irrespective of the immunological triggers $[27,34]$. A similar phenomenon has been observed with corneal epithelial cells, where the electric field was suggested to override chemicological cues to guide migration of the cells [35-36]. Thus, there is potential that this novel Prolene suture system may reduce healing times, whilst improving mechanical integrity of the wound itself.

Clearly, this initial study has been confined to one specific suture product. Since the potential benefits from progressive mechanical contraction and electric charge effects on wound healing emanate from viscoelastic recovery, we suggest that any suture material or wound-healing structure with the potential for viscoelastic activity may enable these attributes to be exploited.

\section{Conclusions}

By subjecting a commercially available suture filament (Ethicon Prolene) to an anneal and tensile creep treatment process, our initial study has demonstrated that the suture can undergo a viscoelastic contraction of $\sim 4 \%$ over $0.1-1000 \mathrm{~h}$ and, following a short recovery time ( $\sim 3 \mathrm{~min})$ to a fixed strain, provides a progressively increasing recovery force of $\sim 0.1-1 \mathrm{~N}$. Although further development is required to achieve optimised processing conditions, we have proposed that this suture treatment may facilitate wound healing through viscoelastically induced mechanotransduction (VIM). In addition, we believe that VIM will be augmented by the release of electric charges through viscoelastic activity and we report on the direct detection of these charges.

By using these treatment conditions on suture samples subsequently immersed within a human skin cell culture, we have found a significant increase in cell motility (meandering index, speed) and migration towards the suture, in comparison with samples that were in annealed-only or as-received condition. These results indicate that a viscoelastically active suture should provide a greater stimulation of the wound healing process and the effect can be expected to continue for the duration of the viscoelastic recovery period.

\section{Acknowledgements}

The authors wish to thank Elizabeth Roberts, (Centre for Atherothrombosis and Metabolic Disease, Hull York Medical School), for invaluable knowledge of operating the LiveCyte system. We are also grateful to Dr Howard Snelling, Physics \& Mathematics Department, for support during the electrometer-based study. This research did not receive any specific grant from funding agencies in the public, commercial or not-for-profit sectors. 


\section{References}

[1] C. Dennis, S. Sethu, S. Nayak, L. Mohan, Y. Morsi, G. Manivasagam. Suture materials - Current and emerging trends. J. Biomed. Mater. Res. A, 104A (2016) 1544-1559.

[2] W. Zhao, L. Liu, F. Zhang, J. Leng, Y. Liu. Shape memory polymers and their composites in biomedical applications. Mater. Sci. Eng. C, 97 (2019) 864-883.

[3] X. Jing, H.Y. Mi, H.X. Huang, L.S. Turng. Shape memory thermoplastic polyurethane (TPU) / poly ( $\varepsilon$-caprolactone) (PCL) blends as self-knotting sutures. J. Mech. Behaviour Biomed. Mater., 64 (2016) 94-103.

[4] R. Duarah, Y.P. Singh, P. Gupta, B.B. Mandal, N. Karak. Smart self-tightening surgical suture from a tough bio-based hyperbranched polyurethane/reduced carbon dot nanocomposite. Biomed. Mater., 13 (2018) 045004.

[5] F. Morelli, A. Anderson, A. McLister, J.J. Fearon, J. Davis. Electrochemically driven reagent release from an electronic suture. Electrochem. Comm. 81 (2017) 70-73.

[6] N. Davies. Smart Sutures. AATCC Review, E-Textiles Series, 17 (2017) 38-43.

[7] A. Lambertz, R. Vogels, D. Busch, P. Schuster, S. Jockenhovel, U. Neumann, U. Klinge, C. Klink. Laparotomy closure using an elastic suture: A promising approach. J. Biomed. Mater. Res. B, Appl. Biomater., 103 (2015) 417-423.

[8] K.S. Fancey. Viscoelastically prestressed polymeric matrix composites: An overview. J Reinf. Plast. Compos., 35 (2016) 1290-1301.

[9] A. Fazal, K.S. Fancey. Viscoelastically generated prestress from ultra-high molecular weight polyethylene fibres. J. Mater. Sci., 48 (2013) 5559-5570.

[10] Y. Qin, K.S. Fancey. Towards "green" viscoelastically prestressed composites: Cellulose fibre reinforcement. Compos. Part B, 154 (2018) 439-448.

[11] B. Wang, K.S. Fancey. A bistable morphing composite using viscoelastically generated prestress. Mater. Lett., 158 (2015) 108-110.

[12] A.M. Esfahani, J. Rosenbohm, K. Reddy, X. Jin, T. Bouzid, B. Riehl, E. Kim, J. Lim, R. Yang. Tissue regeneration from mechanical stretching of cell-cell adhesion. Tissue Eng. Part C, 25 (2019) 631-640.

[13] C. Ge, B. Wang, K.S. Fancey. An evaluation of the scanning electron microscope mirror effect to study viscoelastically prestressed polymeric matrix composites. Mater. Today Comm., 12 (2017) 79-87.

[14] B. Kchaou, C. Turki, M. Salvia, Z. Fakhfakh, D. Treheux. Dielectric and friction behaviour of unidirectional glass fibre reinforced epoxy (GFRE). Wear, 265 (2008) 763-771.

[15] G. Blaise. Charge localization and transport in disordered dielectric materials. J. Electrostat., 50 (2001) 69-89.

[16] G. Blaise, C. Le Gressus. Charging and flashover induced by surface polarization relaxation process. J. Appl. Phys., 69 (1991) 6334-6339.

[17] K.S. Fancey, A. Fazal. Prestressed polymeric matrix composites: Longevity aspects. Polym. Compos., 37 (2016) 2092-2097.

[18] K.S. Fancey. A latch-based Weibull model for polymeric creep and recovery. J. Polym. Eng., 21 (2001) 489-510.

[19] K.S. Fancey. A mechanical model for creep, recovery and stress relaxation in polymeric materials. J. Mater. Sci., 40 (2005) 4827-4831.

[20] J.W.C. Pang, B.M. Lamin, K.S. Fancey. Force measurement from viscoelastically recovering Nylon 6,6 fibres. Mater. Lett., 62 (2008) 1693-1696. 
[21] V.M. Moreno-Villa, M.A. Ponce-Velez, E. Valle-Jaime, J.L. Fierro-Chávez. Effect of surface charge on hydrophobicity levels of insulating materials. IEE Proc. - Gener., Trans. and Dist., 145 (1998) 675-681.

[22] K.S. Fancey. Viscoelastically prestressed composites - where next? 18th European Conference on Composite Materials (ECCM18), Athens, Greece, 24-28th June 2018.

[23] K.S. Fancey, L.A. France. Suture thread products. U.K. patent application W02019175297; international filing date: 14th March 2019.

[24] P.L. Farber, B. Hochman, F. Furtado, L.M. Ferreira. Electricity and colloidal stability: How charge distribution in the tissue can affect wound healing. Medical Hypotheses, 82 (2014) 199 204.

[25] B. Wang, K.S. Fancey. Viscoelastically prestressed polymeric matrix composites: An investigation into fibre deformation and prestress mechanisms. Compos. Part A, 111 (2018) 106104.

[26] R. Kasprowicz, R. Suman, P. O’Toole. Characterising live cell behaviour: Traditional label-free and quantitative phase imaging approaches. Internat. J. Biochem. \& Cell Biol., 84 (2017) 89-95.

[27] A.H. Guo, B. Song, B. Reid, Y. Gu, J.V. Forrester, C.A.B. Jahoder, M. Zhao. Effects of physiological electric fields on migration of human dermal fibroblasts. J. Invest. Dermatol., 130 (2010) 2320-7.

[28] W.S. Ramsey, W. Hertl, E.D. Nowlan, N.J. Binkowski. Surface Treatments and Cell Attachment. In Vitro, 20 (1984) 802-808

[29] T.J. Jeon, R. Gao, H. Kim, A. Lee, P. Jeon, P.N. Devreotes, M. Zhao. Cell migration directionality and speed are independently regulated by RasG and G $\beta$ in Dictyostelium cells in electrotaxis. Biology Open, 8 (2019).

[30] R. Nuccitelli. A role for endogenous electric fields in wound healing. Curr. Top. Dev. Biol., 58 (2003) 1-26.

[31] P. Bainbridge. Wound healing and the role of fibroblasts. J. Wound Care, 22 (2013) 407-408.

[32] L.E. Tracy, R.A. Minasian, E.J. Caterson. Extracellular matrix and dermal fibroblast function in the healing wound. Adv. Wound Care, 3 (2016) 119-136.

[33] L. Maddaluno, C. Urwyler, S. Werner. Fibroblast growth factors: Key players in regeneration and tissue repair. Development (Cambridge), 144 (2017) 4047-4060.

[34] J. Kim, D.H. Kim, K.T. Lim, H. Seonwoo, S.H. Park, Y.R. Kim, Y. Kim, Y.H. Choung, P.H. Choung, J.H. Chung. Charged nanomatrices as efficient platforms for modulating cell adhesion and shape. Tissue Eng. Part C, Methods, 18, (2012) 913-23.

[35] M. Zhao, A. Agius-Fernandez, J.V. Forrester, C.D. McCaig. Orientation and directed migration of cultured corneal epithelial cells in small electric fields are serum dependent. J. Cell Sci., 109 (1996) 1405-1414.

[36] M. Zhao, B. Song, J. Pu, T. Wada, B. Reid, G.P. Tai, F. Wang, A.H. Guo, P. Walczysko, Y. Gu, et al. Electrical signals control wound healing through phosphatidylinositol-3-OH kinase-gamma and PTEN. Nature, 442 (2006) 457-460. 